\title{
Consenso Português para o Diagnóstico e Gestão Clínica da Demência com Corpos de Lewy (PORTUCALE)
}

\author{
Portuguese Consensus on the Diagnosis and \\ Management of Lewy Body Dementia (PORTUCALE)
}

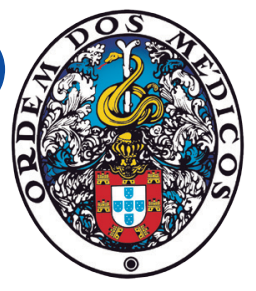

\author{
Ana MONTEIRO ${ }^{1,2}$, Ana Graça VELON ${ }^{3}$, Ana Margarida RODRIGUES ${ }^{4}$, Ana OLIVEIRA ${ }^{5}$, Anabela VALADAS 6 , \\ Camila NÓBREGA',8, Catarina CRUTO' ${ }^{1}$, Dulce NEUTEL ${ }^{9}$, Frederico SIMÕES DO COUTO ${ }^{10}$, Joana MORGADO ${ }^{8,11}$, \\ Joaquim CEREJEIRA' ${ }^{12}$, Luís RUANO ${ }^{13,14,15}$, Miguel GAGO ${ }^{16}$, Miguel GRUNHO ${ }^{17,18}$, Miguel TÁBUAS-PEREIRA ${ }^{19}$, \\ Ricardo TAIPA ${ }^{20}$, Rita MOIRON SIMÕES ${ }^{8,11}$, Rui ARAÚJO 2,21 , Rui BARRETO 6 , Sofia ROCHA ${ }^{4}$, João MASSANO ${ }^{22,21}$ \\ Acta Med Port 2020 Dec;33(12):844-854 - https://doi.org/10.20344/amp.13696
}

\section{RESUMO}

A demência com corpos de Lewy é uma causa comum de demência, provocando a perda progressiva de funções cognitivas e capacidades motoras, alterações comportamentais, e perda de autonomia, com compromisso da qualidade de vida dos doentes e seus familiares. Apesar de ser a segunda causa mais frequente de demência neurodegenerativa, o diagnóstico mantém-se um desafio, devido à sua apresentação clínica heterogénea, sobretudo nas fases iniciais da doença. Por conseguinte, a demência com corpos de Lewy é frequentemente mal diagnosticada e clinicamente gerida de forma insuficiente. A falta de acuidade diagnóstica tem implicações significativas para os doentes, dada a maior suscetibilidade aos efeitos adversos de determinados fármacos, tais como os antipsicóticos, que podem agravar alguns sintomas associados à demência com corpos de Lewy. Por conseguinte, um consenso de especialistas, baseado na análise da literatura mais atual e relevante, e na experiência clínica, é útil para todos os profissionais envolvidos no cuidado destes doentes. O objetivo deste trabalho é informar e gerar recomendações acerca das melhores abordagens diagnóstica e terapêutica da demência com corpos de Lewy em Portugal. Além disso, sugerimos estratégias para aumentar a sensibilização dos médicos, dos decisores políticos e da sociedade em geral em relação a esta doença.

Palavras-chave: Consenso; Demência/diagnóstico; Demência/tratamento; Doença de Corpos de Lewy/diagnóstico; Doença de Corpos de Lewy/tratamento; Portugal

\section{ABSTRACT}

Lewy body dementia is a common cause of dementia leading to the progressive deterioration of cognitive function and motor skills, behavioral changes, and loss of autonomy, impairing the quality of life of patients and their families. Even though it is the second leading cause of neurodegenerative dementia, diagnosis is still challenging, due to its heterogenous clinical presentation, especially in the early stages of the disease. Accordingly, Lewy body dementia is often misdiagnosed and clinically mismanaged. The lack of diagnostic accuracy has important implications for patients, given their increased susceptibility to the adverse effects of certain drugs, such as antipsychotics, which may worsen some symptoms associated with Lewy body dementia. Therefore, a specialist consensus based on the analysis of the most updated and relevant literature, and on clinical experience, is useful to all professionals involved in the care of these patients. This work aims to inform and provide recommendations about the best diagnostic and therapeutic approaches in Lewy body dementia in Portugal. Moreover, we suggest some strategies in order to raise the awareness of physicians, policy makers, and the society at large regarding this disease.

Keywords: Consensus; Dementia/diagnosis; Dementia/therapy; Lewy Body Disease/diagnosis; Lewy Body Disease/therapy; Portugal

\footnotetext{
1. Serviço de Neurologia. Hospital Pedro Hispano. Unidade Local de Saúde de Matosinhos. Matosinhos. Portugal.

2. Departamento de Neurociências Clínicas e Saúde Mental. Faculdade de Medicina. Universidade do Porto. Porto. Portugal.

3. Serviço de Neurologia. Centro Hospitalar de Trás-os-Montes e Alto Douro. Vila Real. Portugal.

4. Serviço de Neurologia. Hospital de Braga. Braga. Portugal.

5. Serviço de Medicina Nuclear. Centro Hospitalar Universitário de São João. Porto. Portugal.

6. Serviço de Neurologia. Departamento Neurociências. Centro Hospitalar Universitário Lisboa Norte. Lisboa. Portugal.

7. Unidade de Diagnóstico e Intervenção. Centro Hospitalar Psiquiátrico de Lisboa. Lisboa. Portugal.

8. Cognition, Dementia and Memory Clinic. Campus Neurológico Sénior. Torres Vedras. Portugal.

9. Unidade de Medicina de Sono. Hospital Cuf Descobertas. Lisboa. Portugal.

10. Instituto de Farmacologia e Neurociências. Faculdade de Medicina, Universidade de Lisboa. Lisboa. Portugal.

11. Serviço de Neurologia. Hospital Beatriz Ângelo. Loures. Portugal.

12. Faculdade de Medicina. Universidade de Coimbra. Coimbra. Portugal.

13. Serviço de Neurologia. Centro Hospitalar de Entre Douro e Vouga. Santa Maria da Feira. Portugal.

14. Departamento de Ciências da Saúde Pública e Forenses e Educação Médica. Faculdade de Medicina. Universidade do Porto. Porto. Portugal.

15. Unidade de Investigação em Epidemiologia (EPIUnit). Instituto de Saúde Pública. Universidade do Porto. Porto. Portugal.

16. Serviço de Neurologia. Hospital da Senhora da Oliveira. Guimarães. Portugal.

17. Serviço de Neurologia. Hospital Garcia de Orta. Almada. Portugal.

18. Centro de Investigação Interdisciplinar Egas Moniz. Monte da Caparica. Portugal.

19. Serviço de Neurologia. Centro Hospitalar e Universitário de Coimbra. Coimbra. Portugal.

20. Unidade de Neuropatologia, Departamento de Neurociências. Centro Hospitalar Universitário do Porto. Porto. Portugal.

21. Serviço de Neurologia. Centro Hospitalar Universitário de São João. Porto. Portugal.

$\bowtie$ Autor correspondente: João Massano. jmassano@med.up.pt

Recebido: 07 de março de 2020 - Aceite: 06 de julho de 2020 | Copyright $\odot$ Ordem dos Médicos 2020
} 


\section{INTRODUÇÃO}

A demência representa um enorme flagelo na sociedade atual e futura. Foi a quinta principal causa de morte a nível global em 2016, causando 2,4 milhões de mortes e afetando 43,8 milhões de pessoas em todo o mundo. ${ }^{1}$ Em Portugal, a prevalência de demência e défice cognitivo ligeiro em pessoas com mais de 55 anos foi estimada entre $5,5 \%-7,7 \%$ em estudos de base populacional. ${ }^{2-4} \mathrm{~A}$ demência com corpos de Lewy (DCLewy) é a segunda causa mais comum de demência de etiologia neurodegenerativa acima dos 65 anos, levando à perda progressiva das capacidades cognitivas e da autonomia, e à diminuição da qualidade de vida dos doentes e seus familiares. ${ }^{5-9} \mathrm{O}$ nome da doença advém da observação de agregados da proteína a-sinucleína, que se acumulam no cérebro (corpos e neurites de Lewy) e se acompanham de perda neuronal. 5,10 As doenças com deposição de $\alpha$-sinucleína, ou sinucleinopatias, incluem a DCLewy, a doença de Parkinson (DP), com eventual desenvolvimento subsequente de demência (demência da doença de Parkinson, DDP) e a atrofia de múltiplos sistemas (AMS). 5,10 A distinção clínica e neuropatológica entre DCLewy e DDP é um desafio e fonte de longa controvérsia. ${ }^{11,12}$

Uma revisão sistemática mostrou uma prevalência média da DCLewy de $0,36 \%$ na população com mais de 65 anos e de $4,2 \%$ na população com demência. ${ }^{6}$ Contudo, estes autores estimaram uma prevalência clínica de 7,5\% na população com demência em cuidados de saúde secundários. ${ }^{6}$ No Reino Unido, em cuidados de saúde secundários, foi estimada uma prevalência de DCLewy de 4,6\% de todos os casos de demência, e nos Estados Unidos o diagnóstico foi identificado em $5,4 \%$ das pessoas $\geq 65$ anos que contrataram seguros de saúde entre 2011 e $2013.7,13$ Relativamente à incidência da DCLewy, Vann Jones e O'Brien estimaram uma incidência anual de $3,8 \%$ na população geral, dentro dos novos casos de demência. ${ }^{6} \mathrm{Em}$ Portugal, a prevalência da DCLewy foi estimada recentemente em $7,0 \%$, incluindo as formas mistas (concomitância com doença de Alzheimer). ${ }^{3} \mathrm{~A}$ literatura atual sugere que a DCLewy poderá estar subdiagnosticada. ${ }^{7}$

Alguns estudos indicam que a DCLewy apresenta uma maior taxa de declínio cognitivo, maior probabilidade de institucionalização e sobrevida menor em comparação com a doença de Alzheimer (DA), ${ }^{8,14-20}$ Foi também associada a taxas mais altas de admissão hospitalar e internamentos de maior duração em relação à DA. ${ }^{21}$ Para além disso, parece haver maior sobrecarga e níveis mais altos de stress nos cuidadores de doentes com DCLewy, com risco aumentado para o desenvolvimento de perturbações psiquiátricas. ${ }^{22} \mathrm{O}$ quarto consenso do Consórcio da DCLewy (Fourth Consensus Report of the DLB Consortium) estabeleceu recomendações para o diagnóstico clínico e patológico da DCLewy. ${ }^{9} \mathrm{O}$ diagnóstico atempado das doenças neurodegenerativas é importante, pois permite proporcionar um aconselhamento mais adequado acerca da doença, bem como gerir mais eficazmente a medicação sintomática e a referenciação para ensaios clínicos. No entanto, o diag- nóstico da DCLewy é difícil, sobretudo nas fases iniciais da doença, visto que pode ter uma apresentação clínica heterogénea, e ser facilmente confundida com outras doenças, incluindo DA e DP..$^{5,10,23}$ Apesar dos esforços para o aumento da sensibilidade dos critérios de diagnóstico, cerca de $20 \%$ dos doentes com DCLewy são erroneamente diagnosticados..$^{24,25}$

\section{OBJETIVOS DO CONSENSO}

Estando definidos internacionalmente os critérios de diagnóstico para a DCLewy não faz sentido propor novos critérios, evidentemente. Todavia, a aplicabilidade prática à realidade portuguesa desses critérios, publicados em revistas científicas dirigidas a públicos específicos, tornam relevante a criação de um consenso de especialistas, tendo em conta as dificuldades que observamos na nossa prática clínica e o provável subdiagnóstico da doença. Este consenso, baseado na análise da literatura mais atual, e na partilha de experiências, foi criado no sentido de informar e gerar recomendações acerca da melhor abordagem diagnóstica e terapêutica da DCLewy em Portugal.

Os objetivos deste consenso são, à luz da realidade portuguesa:

1) alertar e informar os profissionais de saúde, em particular os médicos, sobre as características clínicas da DCLewy, de forma a melhorar a sua identificação precoce e a gestão clínica;

2) aconselhar sobre os meios complementares de diagnóstico mais úteis;

3) fornecer recomendações específicas sobre o tratamento;

4) indicar estratégias para aumentar a consciencialização em relação à DCLewy.

\section{MATERIAL E MÉTODOS}

Este documento resulta de um encontro multidisciplinar realizado a 13 de abril de 2019, em Monte Real (Portugal), que contou com a participação de médicos especialistas de Medicina Nuclear, Neurologia e Psiquiatria com interesse específico e atividade clínica e/ou académica na área das demências e/ou parkinsonismo.

O plano de trabalhos incluiu duas fases principais: a reunião de consenso e a elaboração do artigo. Na primeira procedeu-se à abordagem do estado da arte sobre a DCLewy em termos de critérios de diagnóstico, biomarcadores e desafios atuais no diagnóstico da doença. De modo a enquadrar e estruturar a discussão presencial, foram previamente enviadas aos participantes algumas questões abordando tópicos práticos acerca do diagnóstico e da gestão clínica da DCLewy, bem como sobre a perceção dos especialistas acerca do conhecimento desta doença entre os profissionais de saúde em Portugal. A primeira parte da reunião foi dedicada à discussão global dos vários tópicos propostos previamente, a que se adicionaram outros que os participantes consideraram pertinentes. $\mathrm{Na}$ segunda parte da reunião criaram-se grupos de trabalho 
para discutir várias temáticas: perceção dos especialistas relativamente ao conhecimento da comunidade médica e da sociedade em geral acerca da DCLewy em Portugal; diferenciação na realização do diagnóstico por profissionais adequadamente preparados; gestão clínica dos doentes com DCLewy; problemas frequentes de segurança clínica; e utilização de meios complementares de diagnóstico adequados, bem como desafios práticos no acesso a estes exames. As conclusões foram depois discutidas pela totalidade do grupo de especialistas, alcançando-se consenso sobre os tópicos a abordar no texto do artigo.

A pesquisa de literatura foi efetuada pelos coautores Ana Monteiro e João Massano. Foi feita uma pesquisa na PubMed de acordo com o algoritmo (dementia with lewy bodies OR lewy body dementia) AND (treatment OR therapy OR therapeutics OR diagnosis OR prognosis OR nonpharmacologic OR exercise). Foram lidos os resumos de todos os artigos publicados em Inglês a partir de 1998, uma vez que a maioria dos artigos disponíveis sobre este tópico foram publicados a partir dessa data. Os dois coautores chegaram a um consenso sobre as referências a utilizar na primeira versão do artigo. Nas sucessivas rondas de revisão os outros coautores sugeriram referências adicionais que consideraram pertinentes.

\section{RESULTADOS DE CONSENSO E ORIENTAÇÕES Características clínicas da DCLewy}

Tal como definido no Fourth Consensus Report of the DLB Consortium, ${ }^{9}$ a presença de demência é essencial para o diagnóstico da DCLewy, definida como declínio cognitivo progressivo de gravidade suficiente para interferir nas funções ocupacionais ou sociais normais, ou nas atividades diárias habituais. ${ }^{9}$ As alterações cognitivas típicas incluem defeitos de atenção, funções executivas e processamento visual, mesmo em fases iniciais da doença. ${ }^{9,26}$

As características clínicas nucleares da DCLewy são o parkinsonismo primário (i.e., não devido a medicamentos ou lesões estruturais cerebrais, como por exemplo vasculares), as alucinações visuais recorrentes e precoces, a perturbação do comportamento no sono REM (PCSREM), e as flutuações cognitivas e da vigília (Tabela 1), bastando duas destas características (em adição à demência) para o diagnóstico de DCLewy provável. ${ }^{9}$

O parkinsonismo primário, presente em $85 \%$ ou mais dos doentes com DCLewy, pode incluir bradicinesia, rigidez e tremor de repouso, sintomas que são comuns à DCLewy e à DP. ${ }^{9,11,27} \mathrm{Na}$ DCLewy considera-se que existe parkinsonismo se pelo menos uma das três características es- tiver presente, ao contrário do que sucede na DP, em que é preciso existir bradicinesia e pelo menos um dos outros sintomas. ${ }^{28}$ De acordo com as recomendações do Fourth Consensus, deve ser diagnosticada DCLewy quando a demência ocorre antes ou simultaneamente com o parkinsonismo, e DDP quando a demência surge num contexto de DP já estabelecida clinicamente. ${ }^{9}$

As alucinações visuais recorrentes e complexas são muito frequentes, podendo surgir em até $80 \%$ dos doentes. ${ }^{9}$ São bem formadas, detalhadas e coloridas, geralmente pessoas adultas, crianças ou animais, podendo ainda existir alucinações 'de passagem' (breves e na periferia do campo visual), sensação de 'presença' e ilusões visuais. ${ }^{9} \mathrm{~A}$ combinação de alucinações visuais com disfunção visuo-espacial ajuda a distinguir DCLewy de DA. ${ }^{11}$

A PCSREM caracteriza-se por perda da normal atonia do sono REM e presença de comportamentos motores durante esta fase de sono que traduzem o conteúdo dos sonhos (i.e. dream enactment). ${ }^{29} \mathrm{O}$ seu diagnóstico faz-se com estudo polissonográfico em laboratório. ${ }^{29}$ Esta perturbação pode ocorrer em aproximadamente $80 \%$ dos doentes com DCLewy e pode preceder outras características da doença em anos ou décadas. ${ }^{29-32}$ Uma proporção importante das pessoas com PCSREM desenvolve uma das sinucleinopatias (e.g. DCLewy, DP, atrofia de múltiplos sistemas), podendo sinalizar um estado prodrómico destas doenças. ${ }^{32-34} \mathrm{~A}$ disfunção cognitiva no momento do diagnóstico da PCSREM sinaliza maior risco de desenvolvimento de DCLewy. ${ }^{35}$

As flutuações cognitivas e da atenção na DCLewy podem ocorrer espontaneamente ao longo do mesmo dia. ${ }^{9} \mathrm{As}$ características que sugerem flutuações diurnas na DCLewy incluem: 1) episódios de letargia e sonolência várias vezes por dia apesar de sono suficiente na noite anterior; 2) sestas durante o dia de duas ou mais horas; 3 ) episódios de olhar parado por longos períodos; e 4) episódios de discurso desorganizado, incompreensível ou sem lógica. ${ }^{36}$ As flutuações podem igualmente estar presentes noutros tipos de demências em fases avançadas, pelo que o valor preditivo para DCLewy é superior quando surgem precocemente. ${ }^{9}$

Adicionalmente às características nucleares da DCLewy, existem também características clínicas de suporte para o diagnóstico desta doença: marcada sensibilidade aos antipsicóticos; instabilidade postural; quedas frequentes; síncope ou outros episódios transitórios de alteração da consciência; hipersónia; hiposmia; alucinações noutras modalidades sensoriais; ilusões visuais; apatia; ansiedade;

Tabela 1 - Características nucleares e sinais de alerta da DCLewy

\section{Em doentes com demência}

Um ou mais dos sintomas cardinais de parkinsonismo primário (i.e., não causado por medicamentos ou lesões estruturais do cérebro).

Alucinações visuais recorrentes e precoces no percurso da doença, espontâneas e complexas (bem formadas, coloridas).

PCSREM, que poderá preceder o declínio cognitivo.

Flutuações cognitivas com variações pronunciadas na atenção e vigília no mesmo dia.

Adaptado de McKeith et al, ${ }^{9}$ Jellinger. ${ }^{24}$

DCLewy, demência com corpos de Lewy; PCSREM, perturbação do comportamento no sono REM. 
depressão; e disautonomia (incluindo sintomas de hipotensão ortostática, taquicardia, incontinência urinária e obstipação). ${ }^{9}$

\section{Recomendações - abordagem inicial}

O primeiro passo para o diagnóstico da DCLewy é a colheita da história clínica, obtida com o doente e família/ cuidador, e do exame objetivo, que deve incluir avaliação neurológica e do estado mental. ${ }^{37}$ Uma vez que várias condições clínicas podem explicar os sintomas de demência, recomendamos que sejam rapidamente excluídas (i.e. a nível dos cuidados de saúde primários) possíveis causas tratáveis.

Uma vez que muitos doentes com DCLewy têm também doença vascular cerebral, que contribui para os défices cognitivos, recomendamos a avaliação da presença de fatores de risco vasculares (FRV) modificáveis e sua adequada gestão, de acordo com as recomendações atuais. É também recomendada a pesquisa de hipotensão ortostática na avaliação inicial e ao longo do seguimento, dado a sua frequência na DCLewy, consequências potenciais (e.g., quedas, agravamento das flutuações cognitivas) e impacto na gestão medicamentosa.

Recomenda-se a realização de estudo analítico e de imagem, incluindo: ${ }^{37}$

- Hemograma completo, para excluir anemia.

- Proteína C reativa (PCR).

- Glicemia em jejum, hemoglobina glicada (HbA1c) e perfil lipídico.

- Provas de função hepática e renal, ionograma e glicose séricos.

- Provas de função tiroidea (e.g. TSH e T4 livre).

- Vitamina B12 e folato séricos.

- Teste de rastreio de infeção por Treponema pallidum (sífilis), como por exemplo Treponema pallidum particle agglutination assay (TPPA).

- Análise sumária de urina, para excluir infeção do trato urinário como causa de delirium.

- Rastreio de drogas de abuso na urina (apenas se clinicamente relevante).

- Teste de rastreio do VIH, para excluir a infeção como causadora ou facilitadora de disfunção cognitiva.

- Eletrocardiograma (ECG), por ser recomendável para a escolha da medicação nestes doentes.

- Tomografia computadorizada (TC) crânio-encefálica para excluir lesões estruturais (ex. tumores), sinais sugestivos de hidrocefalia de pressão normal, hematoma subdural e doença vascular cerebral de relevo.

- Em cuidados de saúde secundários/terciários: ressonância magnética (RM) cerebral, se houver alterações em TC cerebral a merecer esclarecimento, ou suspeita de patologia não diagnosticável por este método ou de demência vascular (quando se justifique avaliar lesões vasculares com maior acuidade), ou se a disponibilidade local da RM for elevada (como alternativa primária à TC).
É fundamental obter um historial completo do uso de fármacos e suspender quaisquer medicamentos que possam potencialmente causar alterações cognitivas, tais como antagonistas da dopamina, dopaminérgicos (a avaliar pelo Neurologista assistente), anticolinérgicos, benzodiazepinas, alguns bloqueadores de canais de cálcio (e.g. flunarizina, cinarizina) e anti-histamínicos de primeira geração (e.g., hidroxizina). ${ }^{37}$

Recomendamos que os médicos avaliem a presença das características nucleares da DCLewy que estão listadas na Tabela 1, de acordo com as recomendações presentes no Fourth Consensus Report of the DLB Consortium. ${ }^{9}$

\section{Métodos complementares de diagnóstico}

Têm sido estudados métodos complementares de diagnóstico (MCD) úteis na DCLewy. ${ }^{29,38}$

É recomendada a realização de uma avaliação neuropsicológica por pessoas idóneas e devidamente treinadas, se localmente disponível. Esta avaliação pode demonstrar defeito de atenção, das funções executivas e visuo-perceptivas mesmo em fases iniciais da doença. ${ }^{26} \mathrm{O}$ exame de imagem estrutural (TC ou RM) pode demonstrar uma relativa preservação da região mesial do lobo temporal, embora a presença de atrofia nesta região não exclua DCLewy. ${ }^{39}$

O Fourth Consensus Report of the DLB Consortium definiu os seguintes biomarcadores indicativos de DCLewy ${ }^{9}$ :

- redução, no corpo estriado, da captação de radiofármaco por transportadores de dopamina pré-sinápticos (DaT), recorrendo a tomografia computorizada por emissão de fotão único (single photon emission computed tomography, SPECT) ou tomografia por emissão de positrões (positron emission tomography, PET);

- redução da captação cardíaca de iodo-123 (123|)-metaiodobenzilguanidina (MIBG) observada na cintigrafia; e

- sono REM sem atonia confirmado por polissonografia (PSG). ${ }^{9}$

A imagiologia dos DaT permite avaliar a integridade do sistema nigroestriado in vivo através de SPECT ou PET. ${ }^{40}$ Apesar de a PET oferecer melhor resolução de imagem, a SPECT é menos dispendiosa e está mais amplamente disponível. ${ }^{40} \mathrm{O}$ radiofármaco $\left[{ }^{123} \mathrm{I}\right]-\mathrm{N}$-(3-fluoropropilo)-2 $\beta$ carbometoxi-3 $\beta$-(4-iodofenil) nortropano (123/-FP-CIT) está comercialmente disponível para fins de diagnóstico (DaTSCAN). ${ }^{40,41}$ Segundo uma revisão sistemática por Brigo et al, a técnica ${ }^{123} \mathrm{I}-\mathrm{FP}-\mathrm{CIT}$ SPECT demonstrou sensibilidade e especificidade superiores a $80 \%$ na diferenciação entre DCLewy e demências não-DCLewy, embora não tenha sido realizada confirmação neuropatológica. ${ }^{42} \mathrm{Em}$ estudos com dados neuropatológicos, o ${ }^{123}$-FP-CIT SPECT demonstrou alta sensibilidade no diagnóstico de DCLewy, com baixa percentagem de falsos negativos, sugerindo poder ser um método útil na exclusão de DCLewy em casos de suspeita clínica desta patologia. ${ }^{42,43}$ 
A cintigrafia com ${ }^{123}$ I-MIBG permite o estudo da integridade da inervação simpática pós-ganglionar cardíaca que, ao contrário do SPECT de DaT, não está alterada noutros tipos de demência para além da DCLewy e DDP. ${ }^{38}$ Apesar de ter sido detetada uma sensibilidade similar entre ${ }^{123} \mid$-FP. -CIT SPECT e a cintigrafia miocárdica com ${ }^{123}$ I-MIBG na deteção de DCLewy, a cintigrafia miocárdica poderá ser mais específica para a exclusão de outros tipos de demência, eventualmente com valor superior na diferenciação entre DCLewy e DA comparativamente com a SPECT e RM cerebral. ${ }^{38,44-46} \mathrm{~A}$ combinação da cintigrafia miocárdica ${ }^{123}$ |-MIBG e da ${ }^{123}$ I-FP-CIT SPECT aumenta a especificidade e a sensibilidade no diagnóstico diferencial entre DCLewy e DA, comparativamente à utilização de um só método. ${ }^{47}$

A cintigrafia miocárdica apresenta algumas desvantagens, tais como a grande prevalência, na população, de doenças que poderão interferir com a captação de ${ }^{123} \mid-$ -MIBG (e.g. enfarte do miocárdio, cardiomiopatia dilatada, diabetes mellitus) ${ }^{38,48}$ Para além disso, vários fármacos podem interferir com a captação de ${ }^{123}$ I-FP-CIT e ${ }^{123 \mid-M I B G,}$ mas os fármacos que interferem com a segunda são de uso mais generalizado. ${ }^{38}$ Adicionalmente, este método requer muito tempo de análise, embora tenha sido reportado que as imagens da fase inicial de captação de ${ }^{123}$ |-MIBG podem ser suficientes para o diagnóstico diferencial entre doenças relacionadas com corpos de Lewy e outras causas de parkinsonismo, e entre DCLewy e DA. ${ }^{45,49}$

A suspeita clínica de PCSREM deve ser confirmada por polisssonografia (PSG) de nível I e requer a existência de sono REM sem atonia e a presença de movimentos durante o sono REM, e que os distúrbios do sono não sejam explicáveis por outras condições médicas. ${ }^{29}$ A PSG de nível I inclui eletroencefalograma (EEG), eletro-oculograma, ECG, eletromiograma, medição do fluxo oro-nasal e do esforço respiratório, monitorização da posição corporal e oximetria de pulso..$^{50}$ É realizada em laboratório de sono e tem supervisão constante por um técnico, mas a acessibilidade a este exame é frequentemente difícil em Portugal. ${ }^{50}$ Recentemente, Fernández-Arcos et al verificaram que a PSG com registo de vídeo sincronizado (V-PSG) é importante para diagnosticar a PCSREM e para a exclusão de condições mimetizadoras em doentes com DCLewy com demência ligeira..$^{51}$ Os autores verificaram ainda manifestações comportamentais anormais não apenas no sono REM mas também durante a vigília, na transição da vigília para sono, no sono non-rapid eye movement (NREM) e no despertar. $^{51}$

Quando não é possível realizar a PSG, o diagnóstico de PCSREM provável pode ser feito através de questionários, alguns dos quais já mostraram sensibilidade e especificidade comparáveis à PSG. ${ }^{29}$ Contudo, estes questionários não conseguem distinguir com precisão a PCSREM de outras condições mimetizadoras, como eventos motores durante o sono REM associados a eventos respiratórios como a síndrome de apneia obstrutiva do sono em REM, parassónias do sono NREM ou confusão noturna em doentes com demência, e não estão validados para a população portu- guesa. ${ }^{29}$

É importante referir que a PCSREM é frequentemente sub-diagnosticada devido à falta de conhecimento sobre esta situação, tanto por parte dos doentes como por parte dos clínicos. ${ }^{33,52,53}$ Curiosamente, um estudo mostrou que a ${ }^{123}$-FP-CIT SPECT poderá ser utilizada para identificar um risco aumentado de desenvolvimento de sinucleinopatias clínicas a curto prazo em doentes com PCSREM idiopática. 54

Em determinadas condições, poderá ponderar-se o recurso a biomarcadores rotineiramente usados no diagnóstico da DA. Há estudos que têm sugerido que biomarcadores presentes no líquido céfalo-raquidiano (LCR) poderão ser úteis no diagnóstico diferencial entre DCLewy e DA, especialmente a proteína tau total e a proteína fosfo-tau181, que mostraram uma grande capacidade de discriminação destas doenças, independentemente do seu estádio. ${ }^{55} \mathrm{~A}$ avaliação da a-sinucleína total e oligomérica presentes no LCR poderá ser também útil para o diagnóstico diferencial sobretudo entre a DCLewy e DA. ${ }^{56}$ Adicionalmente, foi também sugerido que um menor uptake do composto $B$ de Pittsburgh marcado com carbono-11 (PiB) e avaliado por PET (PiB-PET) é capaz de distinguir doentes com DCLewy de doentes com DA com uma precisão de $93 \% .{ }^{57}$ Estes biomarcadores devem ser utilizados apenas por profissionais altamente diferenciados e familiarizados com a interpretação dos resultados.

O diagnóstico pré-clínico das sinucleinopatias reveste-se de grande complexidade e de sérias implicações éticas, dadas as incertezas diagnósticas e a inexistência de estratégias para prevenir o aparecimento e a progressão destas doenças, pelo que estes estudos estão apenas indicados no contexto de investigação clínica específica, não sendo de uso rotineiro na prática clínica corrente. Caso surjam alterações das funções cognitivas e/ou motoras em doentes com PCSREM confirmado por PSG de nível I, estes deverão ser orientados para observação em consulta de especialidade, pelo risco elevado de existir uma sinucleinopatia. $^{34}$

\section{Recomendações - diagnóstico da DCLewy}

- Deve ser diagnosticada DCLewy 'provável' quando duas ou mais características nucleares estão presentes num doente com demência, com ou sem a presença de biomarcadores indicativos; ou se apenas uma característica nuclear está presente, associada a um ou mais biomarcadores indicativos, não devendo a doença ser diagnosticada exclusivamente com base em biomarcadores. ${ }^{9}$

- Deve ser diagnosticada DCLewy 'possível' se apenas uma característica nuclear da DCLewy está presente num doente com demência, sem evidência de biomarcadores indicativos; ou se um ou mais biomarcadores indicativos estão presentes sem características nucleares da DCLewy. ${ }^{9}$

- Os MCD devem ser utilizados de acordo com as recomendações do Fourth Consensus Report of the 
DLB Consortium, sempre que haja indicação clínica e de forma atempada no percurso diagnóstico. ${ }^{9}$

\section{Gestão clínica da DCLewy}

A gestão clínica da DCLewy é complexa, requerendo uma abordagem multidisciplinar e a combinação de intervenções farmacológicas e não farmacológicas. ${ }^{9}$ Atualmente não existe cura nem tratamentos modificadores do curso da doença, sendo o tratamento focado nos sintomas cognitivos, neuropsiquiátricos, motores e outros sintomas. . $^{911}$ Uma vez que as flutuações dos níveis de atenção na DCLewy são particularmente difíceis de distinguir de episódios de delirium, é importante identificar eventuais intercorrências sistémicas (e.g. infeções urinárias) sempre que surjam flutuações cognitivas/comportamentais de novo.

Não existe nenhuma medicação formalmente aprovada em Portugal para a DCLewy, pelo que o uso de fármacos é off label. A evidência atual sugere que os inibidores da acetilcolinesterase (IAchE) são úteis no tratamento da DCLewy. Um ensaio aleatorizado mostrou que o donepezilo $(5-10 \mathrm{mg} / \mathrm{dia})$ produz melhorias cognitivas e globais significativas, sendo bem tolerado e clinicamente seguro, benefícios confirmados numa meta-análise recente. ${ }^{58,59} \mathrm{~A}$ rivastigmina (6 - $12 \mathrm{mg} / \mathrm{dia})$ mostrou benefícios significativos na função cognitiva em doentes com DCLewy, sendo também bem tolerada e geralmente segura. ${ }^{60} \mathrm{~A}$ rivastigmina transdérmica $(4,6$ a $13,3 \mathrm{mg} / \mathrm{dia})$ poderá ser uma boa alternativa em doentes com dificuldade de deglutição ou com intolerância aos IAchE por via oral, tendo sido verificado que, em doentes com PDD, a rivastigmina transdérmica causa menos eventos adversos comparativamente à rivastigmina oral. ${ }^{61}$ Os IAchE não devem ser utilizados em doentes com bradicardia ou bloqueio aurículo-ventricular, e após o seu início deve ser vigiada a frequência cardíaca e intervalo PR e QT em ECG. ${ }^{62}$ Os IAchE poderão reduzir substancialmente os sintomas neuropsiquiátricos (incluindo alucinações visuais e delírios). ${ }^{58,60}$ Estudos preliminares apontaram para possíveis benefícios clínicos da memantina (antagonista do recetor N-metil D-aspartato [NMDA]) em doentes com DCLewy e DDP. ${ }^{63}$ Contudo, a evidência dos benefícios clínicos deste fármaco é ainda baixa nestes doentes, sugerindo apenas um pequeno benefício na impressão clínica global, comportamento e humor. ${ }^{64}$

A prescrição de antipsicóticos só deve ser feita se estritamente necessária e com extrema precaução, dado o risco real de agravamento de vários sintomas, como o parkinsonismo (com risco de morte por insuficiência ventilatória devido a rigidez iatrogénica dos músculos torácicos), flutuações cognitivas e disautonomia. ${ }^{11} \mathrm{~A}$ primeira geração de antipsicóticos (e.g. haloperidol, cloropromazina) está contraindicada nestes doentes. ${ }^{11}$ A quetiapina em doses baixas parece segura do ponto de vista motor, mas uma revisão sistemática não demonstrou eficácia comparativamente ao placebo. ${ }^{9,65,66} \mathrm{~A}$ olanzapina está desaconselhada pelo risco de exacerbação do parkinsonismo. ${ }^{67,68}$ Apesar de algum efeito anticolinérgico e da necessidade de frequente monitorização por risco de agranulocitose, a clozapina pode ser considerada na ausência de efeito de outros fármacos (e.g. quetiapina e IAchE), não existindo estudos de eficácia ou segurança na DCLewy. ${ }^{67,68} \mathrm{~A}$ pimavanserina poderá vir a ser uma alternativa, mas não há ensaios clínicos na DCLewy nem está disponível em Portugal. ${ }^{69} \mathrm{O}$ uso de antipsicóticos obriga à monitorização por ECG pelo risco de aumento do intervalo QT, principalmente quando em uso concomitante com IAchE.

Para tratar a depressão, comum na DCLewy, podem ser utilizados inibidores seletivos da recaptação de serotonina (ISRS), inibidores da recaptação de serotonina-noradrenalina (ISRSN) e mirtazapina, de acordo com a tolerabilidade e resposta clínica. ${ }^{9}$ Estes fármacos podem trazer benefícios no controlo do comportamento e na atenção, mas a evidência disponível neste âmbito é de baixa qualidade. ${ }^{70-75}$

Se o parkinsonismo causar incapacidade deve ser tratado apenas com levodopa. Os agonistas dopaminérgicos, os inibidores da monoaminoxidase B (IMAO-B), os anticolinérgicos e a amantadina não devem ser utilizados, pois há um risco significativo de agravamento das alucinações, confusão e psicose. ${ }^{11}$

A melatonina e o clonazepam são úteis no tratamento da PCSREM..$^{29}$ No entanto, o clonazepam poderá agravar os sintomas cognitivos. ${ }^{9} \mathrm{~A}$ melatonina é a opção com meIhor perfil de segurança, mas o seu uso está limitado pelo elevado custo. A trazodona tem efeito na supressão do sono REM e pode ser útil na gestão da insónia intermédia e na alteração do ciclo sono-vigília. ${ }^{76,77}$ No entanto, a trazodona tem como potencial efeito secundário hipotensão ortostática, podendo levar ao agravamento deste sintoma. Os antidepressivos serotoninérgicos (ex. ISRS), podem agravar a PCSREM.

É crucial desenvolver e validar estratégias terapêuticas não farmacológicas em doentes com DCLewy. ${ }^{9} \mathrm{Em} 2018$ foram publicadas duas revisões sistemáticas acerca dos benefícios destas estratégias. Segundo Morrin et al, apesar de alguns estudos suportarem os benefícios de intervenções não farmacológicas em doentes com DCLewy (incluindo terapia electroconvulsiva, estimulação magnética transcraniana repetitiva, estimulação transcraniana por corrente contínua e exercício físico), não há evidência robusta que suporte a sua utilização nestes doentes ${ }^{78}$ Connors et al. reportaram conclusões semelhantes. ${ }^{79}$

\section{Recomendações - intervenções terapêuticas}

Recomendamos as orientações apresentadas nas Tabelas 2 e 3 :

- Deve ser revista toda a medicação do doente, e cessada a prescrição de fármacos desnecessários ou sem indicação clínica sólida. Aplica-se o mesmo princípio a preparações 'naturais', de 'ervanária', produtos homeopáticos e outros que não estão sujeitos a rigoroso controlo e aprovação pelo Infarmed ou pela Agência Europeia do Medicamento.

- Os IAchE são a primeira linha no tratamento da deterioração cognitiva e das alterações de comportamento, flutuações cognitivas e alucinações na 
DCLewy.

- Os sintomas de depressão e ansiedade crónica devem ser tratados com ISRS ou ISRSN em primeira linha.

- Devem ser vigiados sintomas de PCSREM, que podem ser agravados pelo uso de antidepressivos serotoninérgicos.

- O uso de antipsicóticos deve ser evitado. O mais seguro, mas sem evidência robusta de eficácia, é a quetiapina, que deverá ser utilizada na dose mais baixa e apenas durante o tempo mínimo necessário para a redução de sintomas psicóticos incomodativos para o doente/família, e que não tenham cedido com o uso de IAchE; os antipsicóticos não estão indicados para o tratamento da insónia.

- Devem ser evitados outros fármacos com potencial antidopaminérgico, como metoclopramida e cleboprida, por exemplo.

- O parkinsonismo deve ser tratado se causar incapa- cidade e houver perceção de relação risco-benefício favorável da terapêutica. Para este efeito recomenda-se apenas levodopa, iniciada em dose baixa e subindo lentamente de acordo com a resposta clínica (i.e., start low, go slow).

- As alucinações e ilusões não carecem de tratamento farmacológico se não forem incomodativas para o doente/família; caso sejam perturbadoras da qualidade de vida do doente e dos seus cuidadores, e após exclusão de intercorrências agudas, deverão ser tratadas em primeira linha com IAchE; se forem ineficazes ou surgirem sintomas graves que não permitam aguardar pelo efeito IAchE, poderá ser associada quetiapina na dose eficaz mais baixa e durante o menor tempo possível.

- A PCSREM deve ser tratada se incomodativa para o doente/família, devendo ser considerados os riscos de queda e traumatismo do próprio ou do/a companheiro/a que dorme com o doente. Deve ser

Tabela 2 - Gestão clínica da DCLewy: orientações para clínicos

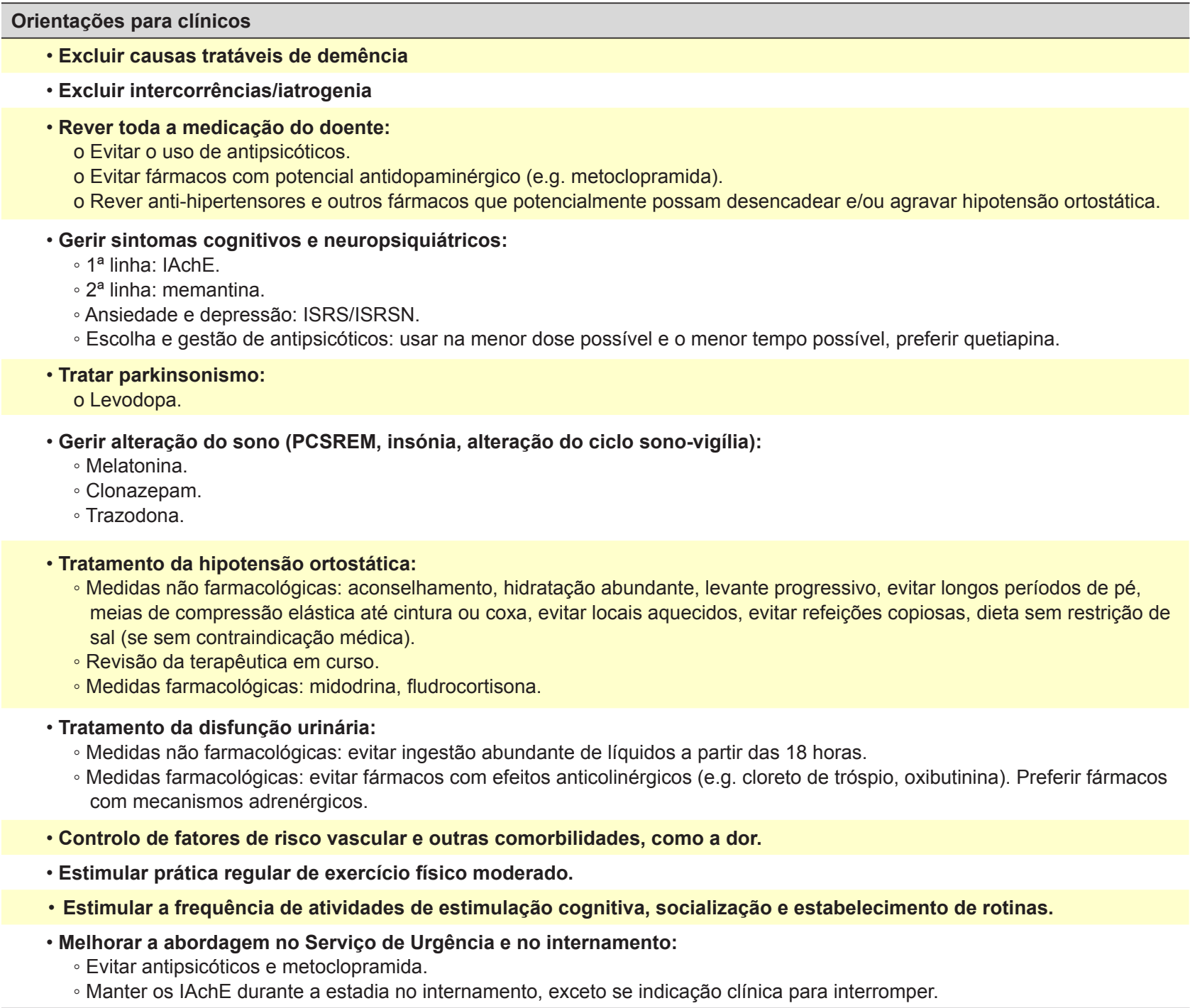

DCLewy: demência com corpos de Lewy; lachE: inibidores da acetilcolinesterase; ISRS_ inibidores seletivos da recaptação da serotonina; ISRSN: inibidores da recaptação da serotonina e noradrenalina; PCSREM: perturbação do comportamento no sono REM 
usada a melatonina (3 - $12 \mathrm{mg}$ ao deitar) ou o clonazepam $(0,25$ - 1,0 mg ao deitar) na dose mínima eficaz. A trazodona (50 - $150 \mathrm{mg}$ ao deitar) poderá ser utilizada para tratamento da PCSREM e para o controlo da insónia inicial e intermédia, e na desregulação do ciclo sono-vigília.

- A hipotensão ortostática deve ser tratada e evitada com medidas não farmacológicas, incluindo hidratação oral, regime de refeições polifracionadas evitando refeições copiosas, levante progressivo da posição de sentado ou deitado, evicção de longos períodos de pé, e uso de meias de compressão elástica. Poderá ser necessário rever a terapêutica em curso e reduzir/suspender a dose de anti-hipertensor.

- Deve ser estimulada a prática regular de exercício físico moderado (e.g. caminhada), durante 30 a 60 minutos diários, pelo menos três a cinco vezes por semana.

- Devem ser incentivadas atividades que promovam a manutenção da funcionalidade, socialização e o estabelecimento de rotinas. As casas devem ser ajustadas às dificuldades da pessoa com demência, devendo estar bem iluminadas, retirando objetos suscetíveis de limitar a locomoção ou aumentar o risco de queda, entre outras medidas.

- Devem ser criadas estruturas apropriadas na comunidade para pessoas com demência e seus cuidadores, incluindo: 1) atividades de socialização, estimulação cognitiva e manutenção da funcionalidade adaptadas ao estádio da doença; 2) apoio aos cuidadores através de grupos de suporte e apoio psicológico; 3) atividades de formação para cuidadores formais e informais; e 4) apoio à investigação.

- Se houver agravamento abrupto dos sintomas da doença, deverá ser considerada e excluída uma causa tratável (e.g. infeção urinária, iatrogenia).

- Em caso de necessidade de internamento por doença aguda, devem ser mantidos os fármacos usados no controlo dos sintomas da doença, como os IAchE e inibidores selectivos da recaptação da serotonina (ISRS), salvo se contraindicação formal pela doença aguda, esperando-se que possam limitar o desenvolvimento de delirium (apesar da pouca evidência nesse sentido) e a utilização consequente de antipsicóticos ou outros fármacos que possam causar efeitos adversos.

- A capacidade de condução está frequentemente afetada na DCLewy, mesmo nas fases iniciais da doença, e deve ser avaliada, de acordo com as disposições legais em vigor, nomeadamente o Decreto-Lei n. ${ }^{\circ}$ 40/2016 (Diário da República, Série I, de 29/Julho). ${ }^{80}$

\section{Consciencialização acerca da DCLewy}

Tal como referido anteriormente, o diagnóstico da DCLewy é difícil, existindo ainda em Portugal uma falta de conhecimento acerca desta doença, que deve ser colmata-
Tabela 3 - Gestão clínica da DCLewy: orientações a abordar com doentes e família

\section{Orientações para doentes e família}

Cessar condução

Educação à família e cuidadores

Vigiar depressão/risco de suicídio

Estimular contacto com associações de doentes

Abordar Regime Jurídico do Maior Acompanhado. ${ }^{a}$

a Lei $n^{\circ} 49 / 2018$, de 14 de agosto. ${ }^{82}$

da, não só nos cuidados de saúde, mas também na sociedade em geral. Para além da importância da diferenciação dos serviços de Neurologia e Psiquiatria na demência, é de salientar que os cuidados de saúde primários e os cuidados continuados na comunidade vão ter um papel cada vez mais importante na DCLewy, tal como noutras doenças neurodegenerativas relacionadas com o envelhecimento. Os doentes com suspeita ou diagnóstico de DCLewy provável ou possível devem ser encaminhados para serviços com especialização em demência, de acordo com os recursos de saúde disponíveis, para confirmação do diagnóstico e definição diferenciada da estratégia terapêutica.

\section{Recomendações - consciencialização para a doença}

A divulgação da DCLewy deve aumentar, não só nos diversos contextos clínicos, mas também na sociedade em geral. Este papel deve ser desempenhado por profissionais de saúde especializados nesta área, sociedades científicas relevantes, entidades governamentais (e.g. Direção-Geral da Saúde) e associações de doentes, preferencialmente de forma concertada e sistemática. Intervenções como as de Susan Williams, que descreve o impressionante caso do brilhante actor e comediante norte-americano Robin Williams, seu marido, contribuem extraordinariamente para a consciencialização acerca da DCLewy. ${ }^{83}$

A formação médica especializada em demências e doenças do movimento (sobretudo parkinsonismo) deve ser reforçada junto das especialidades médicas que, para além da Neurologia e Psiquiatria, terão potencialmente contacto com doentes com DCLewy, em particular a Medicina Geral e Familiar, Medicina Interna, Medicina Paliativa, e Pneumologia.

A oferta da formação pós-graduada em perturbações do sono e PCSREM deve ser aumentada, direcionada a médicos internos e especialistas. Esta deve ser fomentada pela academia, sociedades científicas relevantes, Ordem dos Médicos e serviços clínicos com idoneidade formativa.

Os doentes e familiares devem ser estimulados a criarem as suas associações, que devem ser acarinhadas e ouvidas pelas entidades relevantes.

Com o objetivo de aumentar o conhecimento sobre a DCLewy junto dos cuidadores informais e da sociedade em geral, propomos duas abordagens: 1) a criação de encontros sobre demência, dirigidos à população geral, por entidades idóneas; e 2) a instituição do Dia Nacional da Pessoa com Demência. Este Dia deverá ter um logotipo 
associado, uma data anual fixa, e uma mensagem clara. Os autores deste artigo irão definir uma estratégia para endereçar formalmente estas preocupações e propostas às entidades oficiais com poder legislativo e de decisão política nesta matéria. Será útil que outros interessados tenham também intervenções neste sentido (e.g. associações de doentes, sociedades científicas).

\section{Outras recomendações}

Várias questões fundamentais deverão ser futuramente abordadas de forma consistente em Portugal, tais como as respostas sociais existentes para os doentes com demência, incluindo a DCLewy. Os doentes devem ter acompanhamento adequado na comunidade, bem como cuidados paliativos e de fim de vida específicos, com fortes preocupações humanistas e de acordo com sólidos princípios bioéticos. É fundamental criar estruturas de apoio na comunidade para os cuidadores, de modo a promover o seu bem-estar. Para além disso, é importante fomentar a reflexão e discussão acerca do registo do testamento vital junto de doentes com DCLewy. Assim, o doente poderá expressar atempadamente a sua vontade acerca dos cuidados de saúde que deseja ou não receber quando se encontrar incapaz de o fazer. ${ }^{81}$

Por fim, de modo a melhorar a investigação e os padrões de cuidado clínico dos doentes com DCLewy em Portugal, é necessário sensibilizar os médicos, os doentes e as suas famílias para a importância da doação de cérebros para o Banco Português de Cérebros (http://www.bancodecerebros.chporto.pt/). Esta estrutura permite a recolha e análise dos cérebros dos doentes, sendo crucial para a validação do diagnóstico clínico. Deve ser dado conhecimento às famílias e aos doentes acerca do Banco Português de Cérebros, explicando a importância deste conhecimento e permitindo que os doentes e as suas famílias tomem decisões atempadas acerca desta possibilidade.

\section{AGRADECIMENTOS}

Os autores agradecem a Ana Morgado e Sofia Nunes da Scientific ToolBox Consulting (Lisboa, Portugal) pela prestação de serviços de medical writing, financiados pela GE Healthcare Espanha.

\section{CONFLITOS DE INTERESSE}

Camila Nóbrega exerceu atividade de consultoria para a Tecnifar, Atral Cipan e Baldacci; foi palestrante para as empresas Tecnifar e Novartis; foi patrocinada para a deslocação e inscrição em reuniões científicas pela Bial.

Luís Ruano exerceu atividade de consultoria para as empresas Roche, Biogen e Sanofi; recebeu bolsa de investigação para projeto científico da empresa Biogen; recebeu fundos para a deslocação a reuniões científicas de Novartis, Biogen, Sanofi e Roche.

Miguel Gago, no último ano, recebeu fundos para a deslocação a reuniões científicas de Bial.

Miguel Grunho exerceu atividade de consultoria para as empresas Abbvie, Merck, Novartis e Zambon; foi palestrante para as empresas Bial, Novartis, Sanofi Genzyme e Zambon; recebeu fundos para a deslocação a reuniões científicas de Bial, Biogen, Merck, Novartis, Sanofi Genzyme e Zambon.

Rita Moiron Simões, no último ano, exerceu atividade de consultoria para as empresas Abbvie, Zambon e Bial; foi palestrante para as empresas Bial e Zambon; recebeu fundos para a deslocação a reuniões científicas de Bial e Zambon.

João Massano exerceu atividade de consultoria para as empresas Abbvie, Bial, Merck Sharp \& Dohme e Zambon; foi palestrante para as empresas Bial, Boston Scientific, GE Healthcare e Novartis; recebeu fundos para a deslocação a reuniões científicas de Bial, Boston Scientific, Medtronic, Novartis e Roche.

Ana Monteiro, Ana Graça Velon, Ana Margarida Rodrigues, Ana Oliveira, Anabela Valadas, Catarina Cruto, Dulce Neutel, Frederico Simões do Couto, Joana Morgado, Joaquim Cerejeira, Miguel Tábuas Pereira, Ricardo Taipa, Rui Araújo, Rui Barreto e Sofia Rocha não têm conflitos de interesse a declarar.

\section{FONTES DE FINANCIAMENTO}

Este trabalho foi parcialmente financiado pela GE Healthcare Espanha para apoio à logística da realização da reunião de consenso e para apoio de medical writing no âmbito da preparação deste artigo. A GE Healthcare Espanha não teve qualquer papel no desenho do consenso, recolha, análise e interpretação de literatura, redação do manuscrito, nem na decisão de submeter o artigo para publicação. As opiniões expressas no artigo são da responsabilidade dos autores e não são necessariamente as da GE Healthcare Espanha.

\section{REFERÊNCIAS}

1. Nichols E, Szoeke C, Vollset S, Abbasi N, Abd-Allah F, Abdela J, et al. Global, regional, and national burden of Alzheimer's disease and other dementias, 1990-2016: a systematic analysis for the Global Burden of Disease Study 2016. Lancet Neurol. 2019;18:88-106.

2. Nunes B, Silva R, Cruz VT, Roriz J, Pais J, Silva M. Prevalence and pattern of cognitive impairment in rural and urban populations from Northern Portugal. BMC Neurol. 2010;10:42.

3. Goncalves-Pereira M, Cardoso A, Verdelho A, Alves da Silva J, Caldas de Almeida M, Fernandes A, et al. The prevalence of dementia in a Portuguese community sample: a 10/66 Dementia Research Group study. BMC Geriatr. 2017;17:261.

4. Ruano L, Araujo N, Branco M, Barreto R, Moreira S, Pais R, et al.
Prevalence and causes of cognitive impairment and dementia in a population-based cohort from Northern Portugal. Am J Alzheimers Dis Other Demen. 2019;34:49-56.

5. Outeiro TF, Koss DJ, Erskine D, Walker L, Kurzawa-Akanbi M, Burn $D$, et al. Dementia with Lewy bodies: an update and outlook. Mol Neurodegener. 2019;14:5.

6. Vann Jones S, O'Brien J. The prevalence and incidence of dementia with Lewy bodies: a systematic review of population and clinical studies. Psychol Med. 2014;44:673-83.

7. Kane J, Surendranathan A, Bentley A, Barker S, Taylor J, Thomas A, et al. Clinical prevalence of Lewy body dementia. Alzheimers Res Ther. 2018;10:19 
8. Mueller C, Ballard C, Corbett A, Aarsland D. The prognosis of dementia with Lewy bodies. Lancet Neurol. 2017;16:390-8.

9. McKeith I, Boeve B, Dickson D, Halliday G, Taylor J, Weintraub D, et al. Diagnosis and management of dementia with Lewy bodies: fourth consensus report of the DLB Consortium. Neurology. 2017;89:88-100.

10. Walker Z, Possin K, Boeve B, Aarsland D. Lewy body dementias. Lancet. 2015;386:1683-97.

11. Capouch S, Farlow M, Brosch J. A review of dementia with Lewy bodies' impact, diagnostic criteria and treatment. Neurol Ther. 2018;7:249-63.

12. Walker L, Stefanis L, Attems J. Clinical and neuropathological differences between Parkinson's disease, Parkinson's disease dementia and dementia with Lewy bodies - current issues and future directions. J Neurochem. 2019;150:467-74.

13. Goodman R, Lochner K, Thambisetty M, Wingo T, Posner S, Ling S Prevalence of dementia subtypes in United States Medicare fee-forservice beneficiaries, 2011-2013. Alzheimers Dement. 2017;13:28-37.

14. Kramberger M, Auestad B, Garcia-Ptacek S, Abdelnour C, Olmo J, Walker Z, et al. Long-term cognitive decline in dementia with Lewy bodies in a large multicenter, international cohort. J Alzheimers Dis. 2017;57:787-95.

15. Rongve A, Soennesyn H, Skogseth R, Oesterhus R, Hortobagyi T, Ballard C, et al. Cognitive decline in dementia with Lewy bodies: a 5-year prospective cohort study. BMJ Open. 2016;6:e010357.

16. Bostrom F, Jonsson L, Minthon L, Londos E. Patients with Lewy body dementia use more resources than those with Alzheimer's disease. Int J Geriatr Psychiatry. 2007;22:713-9.

17. Murman D, Kuo S, Powell M, Colenda C. The impact of parkinsonism on costs of care in patients with $A D$ and dementia with Lewy bodies. Neurology. 2003;61:944-9.

18. Williams M, Xiong C, Morris J, Galvin J. Survival and mortality differences between dementia with Lewy bodies vs Alzheimer disease. Neurology. 2006;67:1935-41.

19. Oesterhus R, Soennesyn H, Rongve A, Ballard C, Aarsland D, Vossius C. Long-term mortality in a cohort of home-dwelling elderly with mild Alzheimer's disease and Lewy body dementia. Dement Geriatr Cogn Disord. 2014;38:161-9.

20. Stubendorff K, Hansson O, Minthon L, Londos E. Differences in survival between patients with dementia with Lewy bodies and patients with Alzheimer's disease--measured from a fixed cognitive level. Dement Geriatr Cogn Disord. 2011;32:408-16.

21. Mueller C, Perera G, Rajkumar A, Bhattarai M, Price A, O'Brien J, et al. Hospitalization in people with dementia with Lewy bodies: frequency, duration, and cost implications. Alzheimers Dement. 2018;10:143-52.

22. Svendsboe E, Terum T, Testad I, Aarsland D, Ulstein I, Corbett A, et al. Caregiver burden in family carers of people with dementia with Lewy bodies and Alzheimer's disease. Int J Geriatr Psychiatry. 2016;31:107583.

23. McKeith I, Taylor J, Thomas A, Donaghy P, Kane J. Revisiting DLB diagnosis: a consideration of prodromal DLB and of the diagnostic overlap with Alzheimer disease. J Geriatr Psychiatry Neurol. 2016;29:249-53.

24. Jellinger KA. Dementia with Lewy bodies and Parkinson's diseasedementia: current concepts and controversies. J Neural Transm (Vienna). 2018;125:615-50.

25. Skogseth R, Hortobagyi T, Soennesyn H, Chwiszczuk L, Ffytche D, Rongve A, et al. Accuracy of clinical diagnosis of dementia with Lewy bodies versus neuropathology. J Alzheimers Dis. 2017;59:1139-52.

26. Oda H, Yamamoto Y, Maeda K. Neuropsychological profile of dementia with Lewy bodies. Psychogeriatrics. 2009;9:85-90.

27. Postuma R, Berg D, Stern M, Poewe W, Olanow C, Oertel W, et al MDS clinical diagnostic criteria for Parkinson's disease. Mov Disord. 2015;30:1591-601.

28. Cabreira V, Massano J. Parkinson's disease: clinical review and update. Acta Med Port. 2019;32:661-70.

29. St Louis E, Boeve A, Boeve B. REM sleep behavior disorder in Parkinson's disease and other synucleinopathies. Mov Disord. 2017;32:645-58.

30. Pao W, Boeve B, Ferman T, Lin S, Smith G, Knopman D, et al. Polysomnographic findings in dementia with Lewy bodies. Neurologist. 2013;19:1-6

31. Boeve B, Silber M, Ferman T, Kokmen E, Smith G, Ivnik R, et al. REM sleep behavior disorder and degenerative dementia: an association likely reflecting Lewy body disease. Neurology. 1998;51:363-70.

32. Galbiati A, Verga L, Giora E, Zucconi M, Ferini-Strambi L. The risk of neurodegeneration in REM sleep behavior disorder: A systematic review and meta-analysis of longitudinal studies. Sleep Med Rev. 2019;43:37-
46.

33. Fernandez-Arcos A, Iranzo A, Serradell M, Gaig C, Santamaria J. The clinical phenotype of idiopathic rapid eye movement sleep behavior disorder at presentation: a study in 203 consecutive patients. Sleep. 2016;39:121-32

34. Iranzo A, Tolosa E, Gelpi E, Molinuevo J, Valldeoriola F, Serradell M, et al. Neurodegenerative disease status and post-mortem pathology in idiopathic rapid-eye-movement sleep behaviour disorder: an observational cohort study. Lancet Neurol. 2013;12:443-53.

35. Genier Marchand D, Montplaisir J, Postuma R, Rahayel S, Gagnon J. Detecting the cognitive prodrome of dementia with Lewy bodies: a prospective study of rem sleep behavior disorder. Sleep. 2017;40.

36. Ferman T, Smith G, Boeve B, Ivnik R, Petersen R, Knopman D, et al. DLB fluctuations: specific features that reliably differentiate DLB from AD and normal aging. Neurology. 2004;62:181-7.

37. Lerner A. Dementia with Lewy bodies. BMJ Best Practice. 2018:1-43.

38. Tiraboschi P, Corso A, Guerra U, Nobili F, Piccardo A, Calcagni M, et al. (123) I-2beta-carbomethoxy-3beta-(4-iodophenyl)-N-(3-fluoropropyl) nortropane single photon emission computed tomography and (123) I-metaiodobenzylguanidine myocardial scintigraphy in differentiating dementia with lewy bodies from other dementias: a comparative study. Ann Neurol. 2016;80:368-78

39. Harper L, Fumagalli G, Barkhof F, Scheltens P, O'Brien J, Bouwman F et al. MRI visual rating scales in the diagnosis of dementia: evaluation in 184 post-mortem confirmed cases. Brain. 2016;139:1211-25

40. McCleery J, Morgan S, Bradley K, Noel-Storr A, Ansorge O, Hyde C. Dopamine transporter imaging for the diagnosis of dementia with Lewy bodies. Cochrane Database Syst Rev. 2015;1:CD010633.

41. European Medicines Agency. DaTSCAN: EPAR summary for the public 2011; [consultado 2019 out 28]. Disponível em: https://www.ema.europa. eu/en/documents/overview/datscan-epar-summary-public_en.pdf.

42. Brigo F, Turri G, Tinazzi M. 123I-FP-CIT SPECT in the differential diagnosis between dementia with Lewy bodies and other dementias. J Neurol Sci. 2015;359:161-71.

43. Thomas A, Attems J, Colloby S, O'Brien J, McKeith I, Walker R, et al. Autopsy validation of 123 I-FP-CIT dopaminergic neuroimaging for the diagnosis of DLB. Neurology. 2017;88:276-83.

44. Yoshita M, Arai H, Arai H, Arai T, Asada T, Fujishiro H, et al. Diagnostic accuracy of 123I-meta-iodobenzylguanidine myocardial scintigraphy in dementia with Lewy bodies: a multicenter study. PLoS One. 2015;10:e0120540.

45. Manabe Y, Inui Y, Toyama H, Kosaka K. 123I-metaiodobenzylguanidine myocardial scintigraphy with early images alone is useful for the differential diagnosis of dementia with Lewy bodies. Psychiatry Res Neuroimaging. 2017;261:75-9.

46. Inui $\mathrm{Y}$, Toyama $\mathrm{H}$, Manabe $\mathrm{Y}$, Sarai $\mathrm{M}$, Iwata $\mathrm{N}$. Comparison of (123) I-MIBG myocardial scintigraphy, brain perfusion SPECT, and voxelbased MRI morphometry for distinguishing between dementia with Lewy bodies and Alzheimer's disease. Ann Nucl Med. 2014;28:796-804.

47. Shimizu S, Hirao K, Kanetaka H, Namioka N, Hatanaka H, Hirose D, et al. Utility of the combination of DAT SPECT and MIBG myocardial scintigraphy in differentiating dementia with Lewy bodies from Alzheimer's disease. Eur J Nucl Med Mol Imaging. 2016;43:184-92.

48. Paolillo S, Rengo G, Pagano G, Pellegrino T, Savarese G, Femminella $\mathrm{GD}$, et al. Impact of diabetes on cardiac sympathetic innervation in patients with heart failure: a 123I meta-iodobenzylguanidine (123 MIBG) scintigraphic study. Diabetes Care. 2013;36:2395-401.

49. Shiiba T, Nishii R, Sasaki M, Kihara $Y$, Tsuruta K, Maeda M, et al. Assessment of the efficacy of early phase parameters by (123)I-MIBG dynamic imaging for distinguishing Lewy body-related diseases from Parkinson's syndrome. Ann Nucl Med. 2015;29:149-56.

50. Rundo JV, Downey III R. Polysomnography. In: Handbook of clinical neurology. Elsevier BV. 2019. p. 381-92.

51. Fernandez-Arcos A, Morenas-Rodriguez E, Santamaria J, SanchezValle R, Llado A, Gaig C, et al. Clinical and video-polysomnographic analysis of rapid eye movement sleep behavior disorder and other sleep disturbances in dementia with Lewy bodies. Sleep. 2019;42.

52. Ohayon M, Schenck C. Violent behavior during sleep: prevalence, comorbidity and consequences. Sleep Med. 2010;11:941-6.

53. White C, Hill E, Morrison I, Riha R. Diagnostic delay in REM sleep behavior disorder (RBD). J Clin Sleep Med. 2012;8:133-6.

54. Iranzo A, Santamaria J, Valldeoriola F, Serradell M, Salamero M, Gaig $\mathrm{C}$, et al. Dopamine transporter imaging deficit predicts early transition to synucleinopathy in idiopathic rapid eye movement sleep behavior disorder. Ann Neurol. 2017;82:419-28.

55. Bousiges O, Blanc F. Diagnostic value of cerebro-spinal fluid biomarkers 
in dementia with Lewy bodies. Clin Chim Acta. 2019:490:222-8.

56. van Steenoven I, Majbour N, Vaikath N, Berendse H, van der Flier W, van de Berg W, et al. alpha-Synuclein species as potential cerebrospinal fluid biomarkers for dementia with Lewy bodies. Mov Disord. 2018;33:172433.

57. Kantarci K, Lowe V, Chen Q, Przybelski S, Lesnick T, Schwarz C, et al. beta-Amyloid PET and neuropathology in dementia with Lewy bodies. Neurology. 2020;94:e282-e91.

58. Mori E, Ikeda M, Kosaka K, Donepezil D. Donepezil for dementia with Lewy bodies: a randomized, placebo-controlled trial. Ann Neurol. 2012;72:41-52.

59. Meng Y, Wang P, Song Y, Wang J. Cholinesterase inhibitors and memantine for Parkinson's disease dementia and Lewy body dementia: A meta-analysis. Exp Ther Med. 2019;17:1611-24.

60. McKeith I, Del Ser T, Spano P, Emre M, Wesnes K, Anand R, et al. Efficacy of rivastigmine in dementia with Lewy bodies: a randomised, doubleblind, placebo-controlled international study. Lancet. 2000;356:2031-6.

61. Emre M, Poewe W, De Deyn P, Barone P, Kulisevsky J, Pourcher E, et al. Long-term safety of rivastigmine in parkinson disease dementia: an open-label, randomized study. Clin Neuropharmacol. 2014;37:9-16.

62. Budson A, Solomon P. Chapter 16 - Cholinesterase inhibitors. In: Memory loss, Alzheimer's disease, and dementia: a practical guide for clinicians. Elsevier; 2016. p.160-73.

63. Aarsland D, Ballard C, Walker Z, Bostrom F, Alves G, Kossakowski $\mathrm{K}$, et al. Memantine in patients with Parkinson's disease dementia or dementia with Lewy bodies: a double-blind, placebo-controlled, multicentre trial. Lancet Neurol. 2009;8:613-8.

64. McShane R, Westby M, Roberts E, Minakaran N, Schneider L, Farrimond L, et al. Memantine for dementia. Cochrane Database Syst Rev. 2019;3:CD003154.

65. Maust D, Kim H, Seyfried L, Chiang C, Kavanagh J, Schneider L, et al. Antipsychotics, other psychotropics, and the risk of death in patients with dementia: number needed to harm. JAMA Psychiatry. 2015;72:43845.

66. Desmarais P, Massoud F, Filion J, Nguyen Q, Bajsarowicz P. Quetiapine for psychosis in Parkinson disease and neurodegenerative Parkinsonian disorders: a systematic review. J Geriatr Psychiatry Neurol. 2016;29:22736.

67. Tousi B. Diagnosis and management of cognitive and behavioral changes in dementia with Lewy bodies. Curr Treat Options Neurol. 2017;19:42.

68. Boot B. Comprehensive treatment of dementia with Lewy bodies.
Alzheimers Res Ther. 2015;7:45.

69. Friedman J. A retrospective study of pimavanserin use in a movement disorders clinic. Clin Neuropharmacol. 2017;40:157-9.

70. Pollock B, Mulsant B, Sweet R, Burgio L, Kirshner M, Shuster K, et al. An open pilot study of citalopram for behavioral disturbances of dementia. Plasma levels and real-time observations. Am J Geriatr Psychiatry. 1997;5:70-8.

71. Sultzer D, Gray KF, Gunay I, Berisford M, Mahler M. A double-blind comparison of trazodone and haloperidol for treatment of agitation in patients with dementia. Am J Geriatr Psychiatry. 1997;5:60-9.

72. Pollock B, Mulsant B, Rosen J, Sweet R, Mazumdar S, Bharucha A, et al. Comparison of citalopram, perphenazine, and placebo for the acute treatment of psychosis and behavioral disturbances in hospitalized, demented patients. Am J Psychiatry. 2002;159:460-5.

73. Seitz D, Adunuri N, Gill S, Gruneir A, Herrmann N, Rochon P. Antidepressants for agitation and psychosis in dementia. Cochrane Database Syst Rev. 2011;2:CD008191.

74. Kozman M, Wattis J, Curran S. Pharmacological management of behavioural and psychological disturbance in dementia. Hum Psychopharmacol. 2006;21:1-12.

75. Chow T, Pollock B, Milgram N. Potential cognitive enhancing and disease modification effects of SSRIs for Alzheimer's disease. Neuropsychiatr Dis Treat. 2007;3:627-36.

76. Jaffer K, Chang T, Vanle B, Dang J, Steiner A, Loera N, et al. Trazodone for insomnia: a systematic review. Innov Clin Neurosci. 2017;14:24-34.

77. Ashford J. Treatment of Alzheimer's disease: trazodone, sleep, serotonin, norepinephrine, and future directions. J Alzheimers Dis. 2019;67:923-30.

78. Morrin H, Fang T, Servant D, Aarsland D, Rajkumar A. Systematic review of the efficacy of non-pharmacological interventions in people with Lewy body dementia. Int Psychogeriatr. 2018;30:395-407.

79. Connors M, Quinto L, McKeith I, Brodaty H, Allan L, Bamford C, et al. Non-pharmacological interventions for Lewy body dementia: a systematic review. Psychol Med. 2018;48:1749-58.

80. Decreto-Lei n. ${ }^{\circ}$ 40/2016. Diário da República, I série, n. ${ }^{\circ} 145$ (2016/07/29). p. 2491-555,

81. Lei n. ${ }^{\circ}$ 25/2012. Diário da República, I série, n. 136 (2012/09/16). p. 3728-30.

82. Lei n. ${ }^{\circ}$ 49/2018. Diário da República, I série, n. 156 (2018/08/14). p. 4072-86.

83. Williams SS. The terrorist inside my husband's brain. Neurology. 2016;87:1308-11. 